\title{
Plasma shut-down with fast impurity puff on ASDEX Upgrade.
}

G. Pautasso, C.J. Fuchs, O. Gruber, G. Haas, C.F. Maggi, M. Maraschek, J. Neuhauser, C. Wittmann, E. Wolfrum, M. Beck, P. Cierpka and the ASDEX Upgrade Team

Max-Planck-Institut für Plasmaphysik, EURATOM Association, 85748 Garching, Germany

\section{Introduction}

The fast injection of impurity gas, such as neon, is routinely used to mitigate disruptions in ASDEX Upgrade. The injection is triggered with the locked mode (LM) signal and leads to the onset of mitigated thermal and current quenches within $5 \mathrm{~ms}$. The mitigation effect is due to a further cooling of the plasma with respect to a natural thermal quench. This paper describes (1) the experimental conditions, (2) the plasma response to the injection of impurities, (3) the understanding of the observed phenomena and the motivation to increase the pressure and the quantity of injected gas in future experiments.

\section{Experimental layout}

Valves. The impurity gas is injected into the plasma with two electromagnetic valves, originally built by Dr. Sergej Egorov at the Technical University in St. Petersburg, and modified in the IPP Laboratories. The current in the solenoid of the valve is generated by an array of capacitors charged at voltages of 200-300 V. The valves have an opening and closing time of $2 \mathrm{~ms}$ and remain open for $4-5 \mathrm{~ms}$. The movement of the valve shutter is monitored by a light gauge, which signal is shown in Fig. 1.

The valves have been mostly operating with neon gas up to 5 bar and have been injecting up to 180 mbarl (4.5 $10^{21}$ atoms) of gas. Dedicated experiments have been carried out with 10 and 15 bar. The valves are situated in sector 13 (ASDEX Upgrade consists of 16 sectors) of the tokamak, in the mid-plane, on the low B field side and $1.5 \mathrm{~m}$ away from the plasma edge. The gas expands firstly into a guiding tube, then in the side-port of the machine and finally into the vessel.

Gas flow. During the opening time of 4-5 ms, the valve releases $60-70 \%$ of the gas into the vessel. This quantity is in agreement with the theoretical prediction of the one-dimensional ideal sonic flow of a gas through a nozzle.

We do not have measurements or simulated values of the gas properties outside of the valve in the actual geometry. Nevertheless it is known ${ }^{[1]}$ that the gas may expand and accelerate beyond the sonic velocity. Our experimental observations confirm that the gas travels from the gas valve to the plasma with a supersonic velocity $v_{p} \simeq \sqrt{(k+1) /(k-1)}$ * $v_{s}=1000 \mathrm{~m} / \mathrm{s}$ (with the specific heat ratio $k=1.35$ and $v_{s}=400 \mathrm{~m} / \mathrm{s}$, the sound velocity, for neon) since the first effects of the gas on the plasma are seen 1-2 ms after the opening of the valve.

The gas properties at the plasma surface can be derived assuming mass conservation $\left(v_{s} n_{s} A_{s}=v_{p} n_{p} A_{p}\right.$, with $n$ and $A$ being the density and the area at the sections $p$ and $s$, i.e. at the the plasma surface and at the sonic section) and adiabatic expansion $\left(p_{p}=p_{1}\left(n_{p} / n_{1}\right)^{k} ; p\right.$ indicates the pressure; the index " 1 " indicates the plasma at rest in the valve). The neon reaching the plasma has a density:

$n_{p}=\left(A_{s} / A_{p}\right) \sqrt{(k-1) /(k+1)}(2 /(k+1))^{[1 /(k-1)]} n_{1}=1.710^{21} m^{-3}\left(A_{p}=2 m^{2}\right.$ is assumed) and a pressure $p_{p}=2.710^{-4} p_{1}=1.410^{-3}$ mbar.

A similar result is obtained assuming jet-free expansion from the valve to the plasma. 
According to Ref. [1]: $n_{p}=0.092\left(x / d_{s}\right)^{-2} n_{1} \simeq 0.610^{-5} n_{1}(x=1.5 m$ is assumed).

\section{Measurements of plasma parameters}

The plasma measurements discussed in this section pertain to lower or upper X-point plasmas in the shot range 18600 - 20000, in flat-top, with an elongation larger than 1.5, which underwent a disruption with or without neon puff.

- Density. ASDEX Upgrade is equipped with several diagnostics for the measurement of the electron density. Among them, (1) the CO2 interferometer and (2) the lithium beam diagnostic can record the fast changes of the electron density following the gas puff.

The CO2 interferometer located in sector 11 measures the line integrated electron density along two vertical chords through the plasma core and plasma periphery on the high field side. This interferometer is not subject to fringe jumps during the disruption and provides believable measurements during the whole thermal and current quench.

A database of line integrated densities before $\left(N_{e(b e f o r e)}\right)$ and after $\left(N_{e(\text { after })}\right)$ the disruption reveals a large range of changes of the density during the thermal quench. Figures 2 (a) and (b) show scatter plot of $N_{e(\text { after })}$ versus $N_{e(\text { before })}$ as seen by the channel V-1 and V-2 respectively. $N_{e \text { (before) }}$ is the line integrated density averaged in the time interval $\left[\left(t_{t h . q u .}-20 m s\right)-\left(t_{t h . q u .}-10 m s\right)\right] . N_{e(a f t e r)}$ is averaged in $\left[t_{t h . q u .}-\left(t_{t h . q u .}+5 m s\right)\right]$. $N_{e(a f t e r)} / N_{e(\text { before })}$ seen by channel V-1 has a large standard deviation and is in average 1.5 and 1.8 , respectively for unmitigated and mitigated disruptions. $N_{e(\text { after })} / N_{e(\text { before })}$ seen by channel V-2 is on average of the order of 10, independent of mitigation.

We must conclude that the gas puff does not contribute to a significant increase of the bulk plasma density after the thermal quench. The edge density can increase significantly during the thermal quench independently of the gas puff. The location and the mechanism of this increase cannot be investigated further with these integrated density measurements.

The lithium ( $\mathrm{Li}$ ) beam diagnostic located in sector 9 views horizontal profiles of the plasma edge above the midplane. After the neon puff, it reveals an increase of the electron density in the SOL of 1 order of magnitude within its finest time resolution interval of 1-2 ms. However this diagnostic cannot measure the density profile after the onset of the (natural or induced) thermal quench because the observed LiI emission is dominated by background radiation in this phase.

Theory. These measurements are consistent with elementary theoretical considerations. A neutral beam with a density $n_{0}$ crossing a plasma with electron density $n_{e}$ decays according to the exponential law: $\frac{\partial n_{0}}{\partial x}=-S n_{0} n_{e} / v \equiv-n_{0} /(v \tau)$ where $S$ is the electron impact ionization cross-section ${ }^{[2]}, v$ is the velocity of the neutrals and $\tau$ is the ionization time constant.

According to this equation, neon neutrals are not expected to survive a long way in a tokamak plasma while diffusing from the edge into the center. For shot 19737, for example, $n_{e}=6.510^{18} \mathrm{~m}^{-3}$ and $T_{e}=200 \mathrm{eV}$ at the separatrix before the gas puff, which correspond to $\tau=3 \mu \mathrm{s}$ and to a penetration depth of $2.4 \mathrm{~mm}$ (assuming $v=2 c_{s}=$ $800 \mathrm{~m} / \mathrm{s}$ ). These theoretical predictions are consistent with the measurements.

- Temperature, SXR and MHD activity. Measurements (ECE) of electron temperature profiles show the effect of the impurity on the plasma. Figure 3 illustrates the time history of several ECE channels. The gas valve is opened at $\mathrm{t}=1.310 \mathrm{~s}$ in this case. After $2 \mathrm{~ms}$ the edge channels record a decrease of the temperature. The whole profile narrows in the following $2 \mathrm{~ms}$ without affecting the temperature within the $\rho_{\theta}<0.6$ region. Afterwards the whole temperature profile collapses within $0.1 \mathrm{~ms}$ as in a natural 
thermal quench. Data on electron temperature profiles during a gas puff are rare because (1) the ECE measurements are often in cut-off in pre-disruptive plasmas with a large density or go in cut-off after puffing, and (2) the YAG measurements are still too slow to provide a time history of the temperature profile. The SXR diagnostic shows a similar behavior of the line integrated emission.

The massive injection of impurities triggers the onset of large MHD activity. In the case of a neon puff triggered by the LM, the plasma is not rotating and the structure of the growing fast modes cannot be described with a definable structure or the usual $(\mathrm{m}, \mathrm{n})$ numbers. In the few cases of a neon puff in a healthy plasma, the fast growing mode retains the dominant $(\mathrm{m}, \mathrm{n})$ structure of the small existing rotating pre-disruption mode (3,1: for example).

- Contamination of the machine and runaways. Measurements of concentration of neon were carried out with the CXRS diagnostic tuned on the NeX $(n=11$ rightarrow 10) transition line. The concentration of neon in the discharge after a fast shut-down was below $0.1 \%$. No neon build up was observed when the standard quantity of killer gas ( $180 \mathrm{mbarl}$ ) was fired in subsequent discharges. Concentrations in the same order of magnitude are seen by the Bragg spectrometer. In addition, it has been observed that the plasma performance does not deteriorate after subsequent shut-downs with neon.

Runaway electrons are rare events and appear only in shots with target density $n_{e}<410^{19}$ and $B_{t}>2 T$.

- Mechanical loads. The mechanical forces on the vessel (halo currents and forces on the passive stabilizing loop) are reduced by a factor of 2-3 with respect to their representative values in unmitigated disruptions. A clear correlation between maximum forces on the vessel and maximum vertical excursion of the plasma is found in the database (Fig. 4).

\section{Discussion and conclusion}

The number of impurity atoms injected during the fast shut-down amounts to 50-100\% of the plasma inventory. Nevertheless most of the impurity atoms do not penetrate into the plasma and build up a cooling mantel at the SOL. The resulting disruption is similar to a density-radiation limit disruption with fast narrowing of the temperature profile, peaking of the plasma current (increase of the internal inductance observed) and onset of MHD modes leading to disruption.

The neon content in shots following a fast plasma shut-down amounts to a small fraction of the natural $Z_{\text {eff }}$, does not accumulate and does not influence the performance of the following discharges.

The mitigation of the mechanical forces after impurity injection is robust and largely documented. Dedicated experiments with larger (factor of 3) gas pressure and gas quantity show a further reduction of the forces and motivates the use of stronger impurity puffs. The effect of impurity injection on the thermal loads onto plasma facing components requires a dedicated analysis, which is still underway.

The valve is being developed further to enable operation with a reservoir pressure of 10-50 bars. The modified valve will allow us to investigate further the reduction of forces and heat loads as function of the injected number of atoms and gas pressure.

\section{References.}

[1] H. Pauly, Atom, Molecule and Cluster Beams, Vol. 1, Springer Verlag, 2000, Page 109.

[2] W. Lotz. IPP Report 1/62, May 1967. 

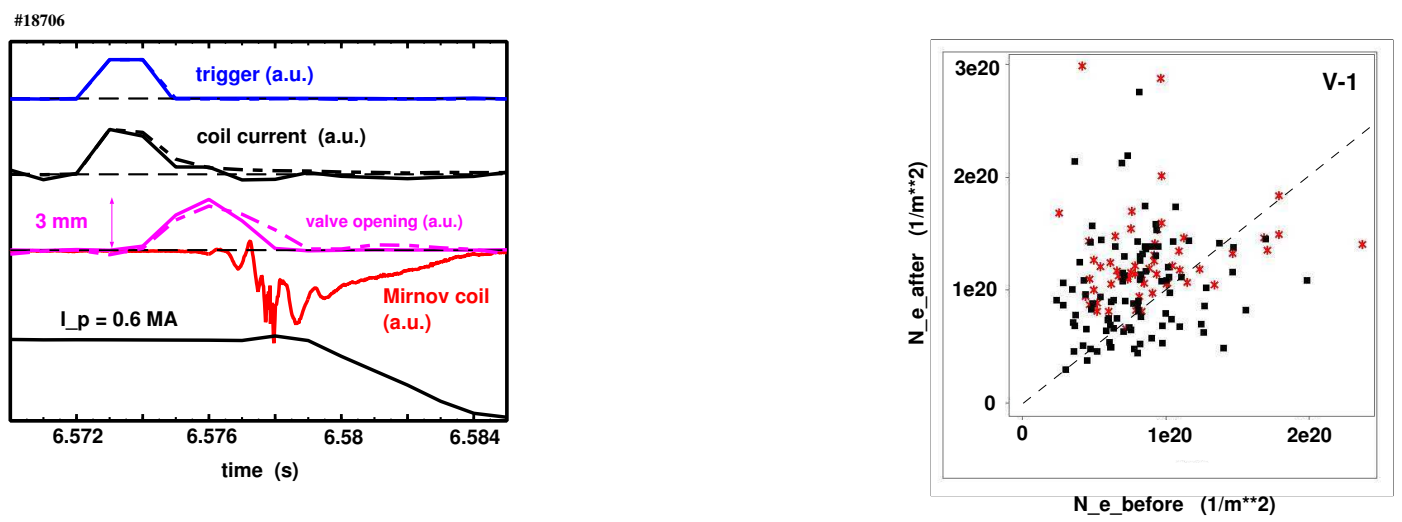

Figure 1. Temporal sequence of events: trigger, opening of the valve and disruption.

Figure 2. After- versus pre-disruption line integrated density seen by the channels V-1 and $\mathrm{V}-2$ of the $\mathrm{CO} 2$ interferometer (mitigated disruptions are indicated with dark symbols).

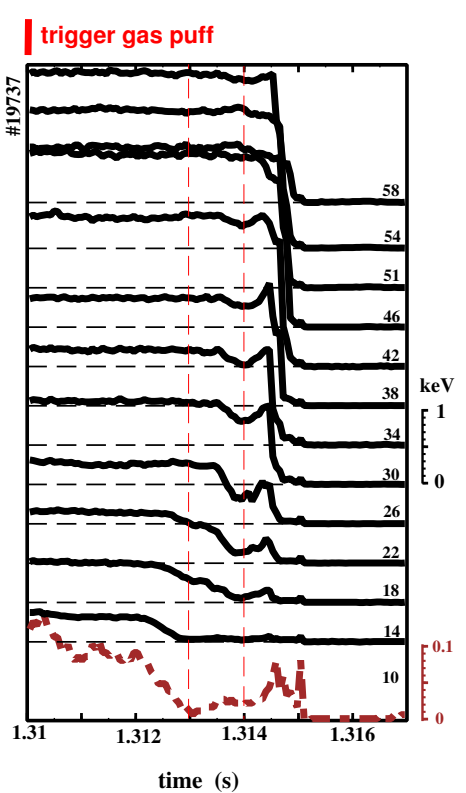

Figure 3. Time evolution of the electron temperature after neon puff (channel numbers increase towards the plasma center).
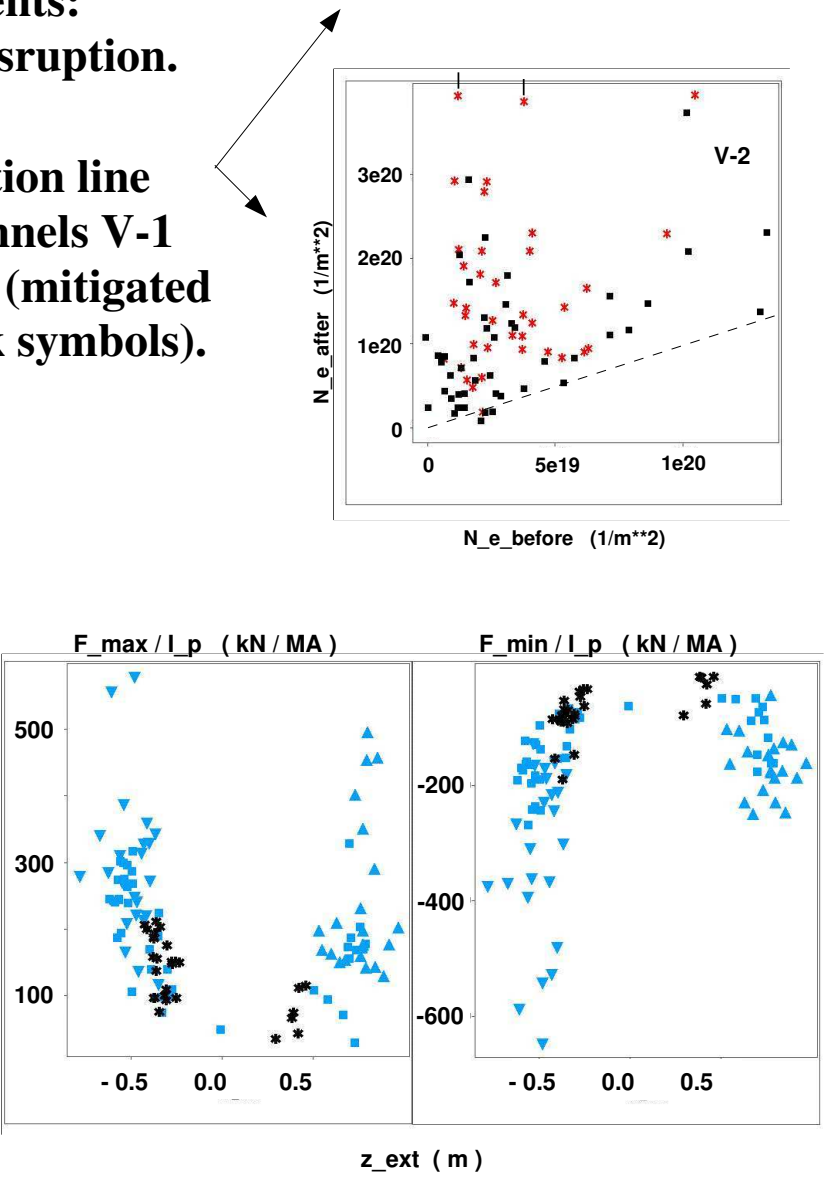

Figure 4. Maximum and minimum forces on the vessel normalized to the plasma current vesus vertical plasma movement. Mitigated disruptions are indicated with dark symbols. 